\title{
Perspectives of Second-Order Blind Identification for Operational Modal Analysis of Civil Structures
}

\author{
C. Rainieri \\ Structural \& Geotechnical Dynamics Lab "StreGa", DiBT Department, University of Molise, \\ Via Duca degli Abruzzi, 86039 Termoli, Italy \\ Correspondence should be addressed to C. Rainieri; carlo.rainieri@unimol.it
}

Received 19 July 2013; Accepted 24 February 2014; Published 11 June 2014

Academic Editor: Nuno Maia

Copyright (C) 2014 C. Rainieri. This is an open access article distributed under the Creative Commons Attribution License, which permits unrestricted use, distribution, and reproduction in any medium, provided the original work is properly cited.

Innovative methods for output-only estimation of the modal properties of civil structures are based on blind source separation techniques. In the present paper attention is focused on the second-order blind identification (SOBI) algorithm and the influence of its analysis parameters on computational time and accuracy of modal parameter estimates. These represent key issues in view of the automation of the algorithm and its integration within vibration-based monitoring systems. The herein reported analyses and results provide useful hints for reduction of computational time and control of accuracy of estimates. The latter topic is of interest in the case of single modal identification tests, too. A criterion for extraction of accurate modal parameter estimates is identified and applied to selected experimental case studies. They are representative of the different levels of complexity that can be encountered during real modal tests. The obtained results point out that SOBI can provide accurate estimates and it can also be automated, confirming that it represents a profitable alternative for output-only modal analysis and vibration-based monitoring of civil structures.

\section{Introduction}

Time domain methods have been proved to be very effective for operational modal analysis (OMA) purposes. They fit a mathematical model to the (correlation functions of the) observed data in order to extract the modal properties [1]. Thus, one of the main problems is related to the optimal setting of the parameters of the model in order to obtain reliable and accurate estimates of the dynamic properties. In practical applications a conservative approach is usually adopted. It is based on the overspecification of the order of the model. This is set large enough to ensure the identification of all physical modes. The amount of overspecification depends on the characteristics of the analyzed dataset. Overmodeling introduces spurious poles besides the physical poles. This makes the modal parameter estimation more complicated. The discrimination between physical and spurious poles is usually based on the construction of the stabilization diagram.

The automatic interpretation of the stabilization diagram is still a challenge. Even if several research efforts have been made to improve its quality (see, for instance, $[2-5]$ ) and to automatically identify the physical poles (see, for instance, [6, $7]$ ), the proposed methods are often computationally intensive and require the computation of a number of parameters and hard and soft validation criteria (see, for instance, [6]); moreover, the reliability of results and the generality of the methods are limited by the need of calibrating thresholds and other analysis parameters for each monitored structure [7]. On the other hand, nonparametric methods do not require the interpretation of stabilization diagrams and, as such, can more easily be automated [8-10].

The use of blind source separation (BSS) techniques in the context of OMA and structural health monitoring (SHM) has been recently proposed [11-15]. BSS techniques extract a set of signals, the so-called sources, from observations of their mixtures [16] based on fairly general assumptions about the sources and the mixing process. BSS techniques can be classified as linear [17] or nonlinear [18], depending on the type of combination of the sources. Moreover, static mixing [17] and convolutive mixing [19] can be considered. 
An increasing number of applications in the field of structural dynamics have recently appeared in the literature $[15,20-22]$. Even if the time response of structures is related to the excitation through a convolutive mixture, the dynamic response of a structure can also be interpreted as a static mixture of sources (the modal coordinates) in the field of modal identification [15]. The physical interpretation of sources as modal coordinates becomes clear by comparing the modal expansion of the dynamic response (1) with the linear and static mixture of sources (2):

$$
\begin{aligned}
& \{y(t)\}=[\Phi]\{q(t)\}, \\
& \{y(t)\}=[A]\{s(t)\} .
\end{aligned}
$$

In fact, the source signals $\{s(t)\}$ play the role of the modal coordinates $\{q(t)\}$ and, on the analogy, there is a one-to-one relationship between the modal matrix $[\Phi]$ and the mixing matrix $[A]$. Thus, in the context of modal identification, BSS techniques aim at recovering the mixing matrix $[A]$, holding the information about the mode shapes, and the modal responses, here represented by the sources $\{s(t)\}$, from their observed mixtures $\{y(t)\}$.

No mathematical model is assumed to describe the process that produced the measured data. The mixing model is the only assumption. Thus, BSS techniques can be referred to as time domain, nonparametric methods for OMA. The absence of stabilization charts to be interpreted or model parameters to be set simplifies the automation and makes BSS techniques an interesting alternative to the classical time domain modal identification methods. Nevertheless, the use of BSS techniques in structural dynamics is still a challenge [14], and a performance assessment of BSS techniques in the context of output-only modal identification of civil structures is certainly of interest.

Some limits in the use of BSS for OMA can be traced back to the basic assumptions of the different techniques, which show different degree of compatibility with the dynamic systems of interest. The applicability of principal component analysis (PCA) to real case studies has been limited by the need for information about the mass matrix [15]. Independent component analysis (ICA) has been recognized to be much more suitable than PCA to vibration data processing for modal identification purposes [15]. The main drawbacks are related to the use of high-order statistics, whose computation is time consuming and difficult in the presence of a scarcity of data, and to the poor modal identification performance of the method for systems characterized by damping ratios larger than $1 \%$ [15]. Methods based on second-order statistics of the observed signals assume that the sources are uncorrelated for all delays and have different spectra. Among these, the second-order blind identification (SOBI) algorithm [17] has been recognized as a promising alternative for modal parameter identification in a number of studies [20-22]. When SOBI is applied to vibration data, the real valued mixing matrix implies real valued mode shape estimates. This can be a drawback of the method in the presence of complex modes. However, taking into account that the basic assumptions of SOBI fit the needs of the output-only identification of dynamics systems and that civil engineering structures often show real modes, in the following sections attention is focused on SOBI and its performance for OMA of civil structures.

After a discussion about how SOBI can be adapted to the analysis of random responses in the context of OMA, the present study investigates the influence of the analysis parameters on accuracy of modal parameter estimates and computational time. The identification performance of SOBI is also assessed against a number of experimental case studies related to civil structures and characterized by increasing degree of complexity. The final objective is the definition of rules of thumbs for the application and automation of SOBI in the context of OMA and vibration-based SHM of civil structures.

\section{SOBI and Modal Identification}

The basics of SOBI are briefly reported in this section to highlight, at the end, how the method has been herein adapted to the analysis of random responses for OMA purposes. This discussion puts in evidence the analysis parameters of the algorithm. Their influence on the quality of modal parameter estimates is investigated in the next section.

When some (additive) noise $\{n(t)\}$ is present in the measured response, (2) can be rewritten as

$$
\{y(t)\}=[A]\{s(t)\}+\{n(t)\} .
$$

The $l$ recorded time histories are, therefore, modelled as a linear combination of $N_{m}$ modal responses (the sources) plus noise. As a consequence, if there are $N_{m}$ modes in the frequency range under investigation, they can be identified only if $\operatorname{rank}([A])=N_{m}$. Since the mixing matrix has dimension $l \times N_{m}$, this implies that the number of measurement channels has to be larger than or equal to the number of active modes: $l \geq N_{m}$. Thus, SOBI can be classified as a loworder method for OMA. Moreover, since the columns of the mixing matrix represent estimates of the mode shapes of the structure under test, a judicious choice of sensor locations is needed so that the observed mode shape vectors are linearly independent and the rank of $[A]$ is preserved.

The issues related to the identifiability of a limited number of modes can be mitigated through the repeated application of band-pass filtering until the entire frequency range of interest is investigated. However, this procedure leads to a major increase in the time of analysis.

A fundamental assumption in SOBI is that the sources are stationary, uncorrelated, and scaled to have unit variance, so their covariance matrix is the identity matrix:

$$
\left[R_{s s}(0)\right]=E\left\{\{s(t)\}\{s(t)\}^{T}\right\}=[I],
$$

where the superscript $T$ denotes transpose. The additive noise is assumed to be a temporally and spatially white stationary random process, with

$$
\begin{gathered}
E[\{n(t)\}]=\{0\}, \\
{\left[R_{n n}(0)\right]=E\left[\{n(t)\}\{n(t)\}^{T}\right]=\sigma^{2}[I] .}
\end{gathered}
$$


If the added noise is also independent of the source signals, this implies

$$
E\left[\{n(t)\}\{s(t)\}^{T}\right]=[0] .
$$

The first step of the algorithm consists of whitening the signal part of the observed data $\{x(t)\}=[A]\{s(t)\}$. This is achieved by applying a linear transformation to $\{x(t)\}$ such that the whitened data $\{z(t)\}$ are uncorrelated and have unit variance:

$$
\begin{aligned}
\{z(t)\} & =[W]\{x(t)\} \Longrightarrow\left[R_{z z}(0)\right] \\
& =E\left[\{z(t)\}\{z(t)\}^{T}\right]=[I] .
\end{aligned}
$$

The matrix $[W]$ defining this transformation is referred to as the whitening matrix. From (8) and (4) it is easy to check that

$$
\begin{aligned}
{\left[R_{z z}(0)\right] } & =[W][A] E\left[\{s(t)\}\{s(t)\}^{T}\right][A]^{T}[W]^{T} \\
& =[W][A][A]^{T}[W]^{T}=[I] .
\end{aligned}
$$

Thus, if $[W]$ is a whitening matrix, $\left[A^{\prime}\right]=[W][A]$ is an $N_{m} \times N_{m}$ unitary matrix. As a consequence, the mixing matrix can be factored as the product of the inverse of the whitening matrix and a unitary matrix (to be determined).

Whitening of the measured response $\{y(t)\}$ also obeys a linear model:

$$
\begin{aligned}
{[W]\{y(t)\} } & =[W]([A]\{s(t)\}+\{n(t)\}) \\
& =\left[A^{\prime}\right]\{s(t)\}+[W]\{n(t)\} .
\end{aligned}
$$

From the covariance matrix of the observed mixture

$$
\left[R_{y y}(0)\right]=E\left[\{y(t)\}\{y(t)\}^{T}\right]=[A][A]^{T}+\sigma^{2}[I],
$$

the following equation is obtained:

$$
[A][A]^{T}=\left[R_{y y}(0)\right]-\sigma^{2}[I] .
$$

Combining (10) with (12) and taking into account (4), (6), and (7), it is possible to show that the whitening matrix $[W]$ can be derived from the covariance matrix $\left[R_{y y}(0)\right]$, provided that the noise covariance matrix is known or can be estimated.

From a practical point of view, once the measured data have been centralized by removal of the mean value from each component of $\{y(t)\}$, whitening is obtained as follows. First of all, the eigenvalue decomposition of $\left[R_{y y}(0)\right]$ is computed:

$$
\left[R_{y y}(0)\right]=E\left[\{y(t)\}\{y(t)\}^{T}\right]=[V][D][V]^{T},
$$

where $[V]$ is the matrix of eigenvectors and $[D]$ is the diagonal matrix of eigenvalues. If only the $N_{m}$ largest eigenvalues $d_{1}, \ldots, d_{N_{m}}$ and the corresponding eigenvectors $\left\{v_{1}\right\}, \ldots,\left\{v_{N_{m}}\right\}$ of $\left[R_{y y}(0)\right]$ are retained, the average of the remaining $l-N_{m}$ eigenvalues yields an estimate $\sigma^{2}$ of the noise variance, under the assumption of white noise [17]. The whitened signals are then computed from the largest eigenvalues and the corresponding eigenvectors as

$$
\begin{aligned}
\{z(t)\} & =\left(\left[D_{N_{m}}\right]-\sigma^{2}\left[I_{N_{m}}\right]\right)^{-1 / 2}\left[V_{N_{m}}\right]^{T}\{y(t)\} \\
& =[W]\{y(t)\},
\end{aligned}
$$

where $\left[D_{N_{m}}\right]$ is the submatrix of $[D]$ holding only the $N_{m}$ largest eigenvalues, $\left[V_{N_{m}}\right]$ is the submatrix of $[V]$ collecting the eigenvectors corresponding to the $N_{m}$ largest eigenvalues of $\left[R_{y y}(0)\right]$, and the whitening matrix is given by

$$
[W]=\left(\left[D_{N_{m}}\right]-\sigma^{2}[I]\right)^{-1 / 2}\left[V_{N_{m}}\right]^{T} .
$$

In (6) the noise variance is assumed to be the same for all channels in agreement with the original formulation of the method [17]. However, in practical applications the noise distribution can vary from sensor to sensor, even if it is typically assumed that two sensors show uncorrelated noise (see, for instance, [23]). Moreover, sensor noise is often $1 / f$ type noise rather than temporally white noise. The white noise assumption is needed to get an estimate $\sigma^{2}$ of the noise variance as the average of the smallest $l-N_{m}$ eigenvalues of $\left[R_{y y}(0)\right]$; see (14), (15), and (16). However, as clarified at the end of this section, the estimation of the noise variance before the extraction of the sources can be avoided for OMA purposes. In fact, SOBI is applied to estimate the correlation functions of both the sources and the noise. The discrimination between modal responses and noise is carried out in a second stage. Thus, the different noise distributions do not affect the modal identification performance of the method in practical applications. The assumption of spatially white noise is not a limiting factor in the context of OMA. However, from a general point of view, the blind identification problem when $\left[R_{n n}(0)\right]$ is a full matrix can be solved by carrying out a robust whitening, based on the decomposition of a linear combination of a set of covariance matrices taken at nonzero time lags [24], instead of the classical whitening given by (13) and (15).

Once the whitened signals have been obtained, the following $p$ time-shifted covariance matrices have to be computed:

$$
\left[R_{z z}\left(\tau_{k}\right)\right], \quad k=1, \ldots, p .
$$

In order to estimate the sources and the mixing matrix, SOBI carries out an approximate joint diagonalization of those $p$ time-shifted covariance matrices according to the joint approximate diagonalization (JAD) technique [17]. The objective of the JAD is to find the unitary matrix [ $\Psi]$ that approximately diagonalizes the time-shifted covariance matrices. An optimization problem is defined with respect to the matrix $[\Psi]$ that minimizes the sum of all off-diagonal terms of $[\Psi]^{T}\left[R_{z z}\left(\tau_{k}\right)\right][\Psi](k=1, \ldots, p)$ for the $p$ timeshifted covariance matrices:

$$
\min _{[\Psi]} \sum_{k=1}^{p} \operatorname{off}\left([\Psi]^{T}\left[R_{z z}\left(\tau_{k}\right)\right][\Psi]\right) .
$$

The solution to the minimization problem is found by means of a numerical algorithm based on the Jacobi rotation technique [17]. Two parameters have to be set: the number $p$ of time-shifted covariance matrices to be jointly diagonalized and the threshold $t$ used to stop JAD. Concerning the threshold $t$, the problem of its setting has been analyzed in [25], showing that very small values for $t$ have no sense 
because the diagonality criterion is approximate itself. Thus, it is usually unnecessary to push the accuracy of the rotation matrix to the machine precision. Setting the value of $t$ to the square root of the machine precision is sufficient. Concerning the number $p$ of time-shifted covariance matrices, the diagonalization performance improves when $p$ increases and it rapidly converges in most cases [17]. Once the matrix [ $\Psi$ ] has been obtained, the demixing matrix $[U]$ and the mixing matrix $[A]$ can be computed:

$$
\begin{aligned}
& {[U]=[\Psi]^{T}[W],} \\
& {[A]=[W]^{+}[\Psi],}
\end{aligned}
$$

where the superscript + denotes pseudoinverse. The resulting sources are shift-uncorrelated because the matrices $\left[R_{s s}\left(\tau_{k}\right)\right]$ are nearly diagonal. The sources are obtained as follows:

$$
\{s(t)\}=[U]\{y(t)\} .
$$

The mode shapes of the structure are obtained from the columns of the mixing matrix. The technique for the estimation of natural frequencies and damping ratios depends on the type of data used for modal identification. In the literature SOBI has been applied to free responses, impulse responses, and responses to random excitation [20-22]. In the first two cases, the estimation of natural frequencies and damping ratios from the obtained sources is straightforward. In fact, taking into account that the free vibration response can be expressed as a sum of exponentially decaying sinusoids, fitting this expression to the data allows the estimation of the modal parameters (refer to [22] for more details). In the case of response to random excitation, while the estimation of natural frequencies from the identified sources is again straightforward, this is not the case of damping ratios, whose estimation requires the knowledge of the applied random excitation [20]. However, the extension of SOBI to the analysis of random responses for the estimation of modal parameters including damping is immediate by recognizing that also the correlation function can be expressed as a sum of decaying sinusoids. This is the same basic assumption adopted by the NExT-type procedures [1]. In this case, there is no need to recover the sources, because natural frequencies and damping ratios can be directly estimated from their correlations $R_{s s}\left(\tau_{k}\right)$ as obtained from the JAD.

A simplified approach to the estimation of the modal properties by SOBI in the case of random response can be outlined as follows.

(i) Compute the whitening matrix $[W]$ from the centralized dataset according to (13) and (15); since the number of modes is not known a priori and the criterion for appropriate selection of the number $N_{m}$ of eigenvalues to be retained is still debated, it is possible to set $N_{m}=l$; thus, $l$ sources are extracted from the data even if the number of modes is likely lower than l; as a result, the sources associated with the actual structural modes have to be selected in a second stage based, for instance, on the error in fitting the correlations $R_{s s}\left(\tau_{k}\right)$ with exponentially damped harmonic functions; this approach was originally proposed in [20] for the analysis of impulse responses.

(ii) Compute the whitened dataset $\{z(t)\}$.

(iii) Compute the time-shifted covariance matrices $\left[R_{z z}\left(\tau_{k}\right)\right]$ and select $p$ of them to apply the JAD.

(iv) The JAD of the $p$ time-shifted covariance matrices $\left[R_{z z}\left(\tau_{k}\right)\right]$ with $k=1, \ldots, p$ provides the unitary matrix $[\Psi]$, which allows the computation of the mixing matrix $[A]$ according to (19) and the autocorrelations $R_{s s}\left(\tau_{k}\right)$;

(v) Natural frequencies and damping ratios are finally estimated; different approaches can be adopted, such as regression on zero-crossing times and logarithmic decrement, or single-degree-of-freedom (SDOF) curve fitting estimators; the use of SDOF curve fitting estimators allows the computation of the fitting error; this makes a quantitative selection of the correlations associated with actual structural modes in the absence of noise rejection in the computation of the whitening matrix possible; the selection of the number of time lags $p$ now plays a primary role in the process, since it not only is responsible for the matrix $[\Psi]$ but also defines the length of the correlation functions $R_{s s}\left(\tau_{k}\right)$; thus, taking into account the physics of the problem, $p$ has to be set as a function of the period of the fundamental mode so that a sufficient number of cycles are present in the correlation of the source associated with the fundamental mode; a criterion for setting of $p$ is given in Section 3.

(vi) The mode shapes are obtained from the columns of the mixing matrix corresponding to the source correlations selected in the previous step of analysis.

It is interesting to note that, unlike the other two-stage modal identification methods, SOBI provides the mixing matrix and, therefore, the mode shape estimates, first; natural frequencies and damping ratios are obtained in a second stage through postprocessing of the obtained sources. Finally, it is worth pointing out that SOBI can identify distinct, eventually closely spaced modes but it shows serious limitations in the presence of repeated modes [26].

\section{Performance Assessment of SOBI for OMA of Civil Structures}

In this section the effect of the parameters $p$ and $t$ on accuracy of modal parameter estimates and computational time of SOBI is investigated. SOBI is applied to OMA of selected case studies. Both simulated and real datasets have been considered. The final objective is the definition of rules of thumbs for the application and automation of SOBI for output-only modal analysis and vibration-based SHM of civil structures. The problem of the definition of criteria and approaches to reject the noise and, therefore, define in advance the expected number of modes is out of the scope of the present paper. SOBI is herein applied in order to extract 


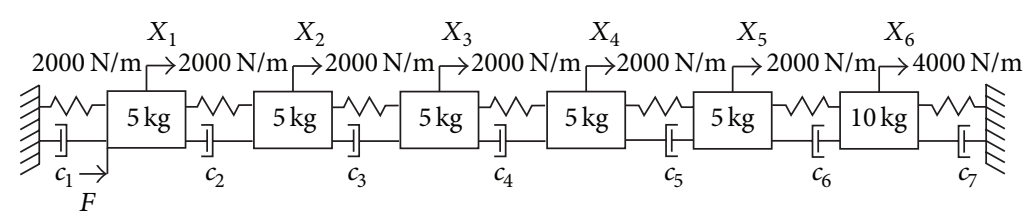

FIgURE 1: The benchmark 6-DOF system.

a number of modes equal to the number of measurement channels, as mentioned in the previous section. The obtained source correlations can represent both modal responses and noise sources. However, noise sources are distinguishable from modal responses [20] (this makes the definition of noise rejection mechanisms less critical in SOBI with respect to parametric time domain modal identification methods, which try to fit a model to the measured system response) and they can be eliminated in the second stage of the analysis aimed at estimating the natural frequencies and damping ratios. After the selection of the sources associated with modal responses, in this study the natural frequency of the $i$ th mode is estimated by a linear regression on the zerocrossing times of the associated correlation function $R_{s s}\left(\tau_{k}\right)$, while damping ratio is calculated through the logarithmic decrement technique on the analogy with another wellestablished OMA technique, the enhanced frequency domain decomposition (EFDD) [27].

Sensitivity analyses have been carried out in order to assess the effect of the parameters governing the JAD (the number $p$ of time-shifted covariance matrices to be jointly diagonalized and the threshold $t$ used to stop the numerical procedure) on accuracy and computational time of SOBI when it is applied to output-only modal analysis. The first analyzed record is represented by the simulated response to a Gaussian white noise applied to degree-of-freedom (DOF) number 1 of the 6-DOF system shown in Figure 1. Rayleigh damping is adopted. Assuming 1\% damping for the first and the last mode, the following proportionality constants have been obtained: $a_{0}=0.1523$ for the mass matrix and $a_{1}=$ $4.15 E-4$ for the stiffness matrix. The modal properties of the system are reported in Table 1 . The response of the system has been simulated at all the six DOFs and it has been sampled at $100 \mathrm{~Hz}$ and decimated at $10 \mathrm{~Hz}$ before processing. White noise has also been added to the time series in order to simulate measurement noise. A signal-to-noise ratio of $5 \mathrm{~dB}$ has been considered. The adopted sampling frequency after decimation results in the extraction of four modal responses and two noise sources.

The results obtained from the application of SOBI to the simulated data have then been validated against operational response measurements of a number of real civil structures. Good quality data from a reinforced concrete structure characterized by well-separated modes (the Tower of the Nations in Naples [28]) and a reinforced concrete structure characterized by two closely spaced modes (the School of Engineering Main Building in Naples [29]) and noisy data from a masonry bell tower characterized by two closely spaced modes (S. Maria del Carmine Bell Tower in Naples [30]) are the considered benchmark record. They have
TABLE 1: Modal properties of the simulated 6-DOF system.

\begin{tabular}{lcc}
\hline Mode number & Natural frequency $[\mathrm{Hz}]$ & Damping ratio [\%] \\
\hline I & 1.509 & 1.00 \\
II & 2.823 & 0.80 \\
III & 3.810 & 0.81 \\
IV & 4.737 & 0.87 \\
V & 5.593 & 0.95 \\
VI & 6.167 & 1.00 \\
\hline
\end{tabular}

been used also to validate an innovative automated OMA procedure in [9]. The selected real test cases are representative of modal identification problems typically encountered in civil engineering and characterized by different degree of difficulty. Reference modal parameters have been extracted from these records by well-established techniques, such as frequency domain decomposition (FDD) [31] and stochastic subspace identification (SSI) $[1,32]$, which have provided very consistent estimates.

The results of the sensitivity analyses on the simulated dataset are presented first. According to these results, some rules of thumbs for the application of SOBI to modal identification are defined and applied to the real case studies. Validation of the effectiveness of the proposed criterion for the selection of $p$ is based on comparisons with the modal estimates provided by FDD and SSI. Even if SOBI and its variants have already been applied to the modal identification of real civil structures [33], this study represents an interesting example of systematic application of SOBI to modal identification problems typically encountered in civil engineering and characterized by different degree of difficulty.

Concerning parameter settings, a careful literature review has provided a recommended value for $t$, as discussed in the previous section. However, this value has been determined according to a theoretical framework and not in view of the application of SOBI to output-only modal analysis and vibration-based SHM. Even if the computational time might not be a problem for single modal identification tests, its control can be of interest for continuous, automated monitoring. The computational time can range from fractions of seconds to several minutes or hours depending on the settings of $t$ and $p$ and the number of measurement channels. Figure 2 shows the dependence of the computational time from $p$ and $t$ for a real dataset consisting of 12 measurement channels. As expected, the larger the number of measurement channels, the higher the computational time of the algorithm, and it increases when $p$ becomes larger and $t$ decreases. 


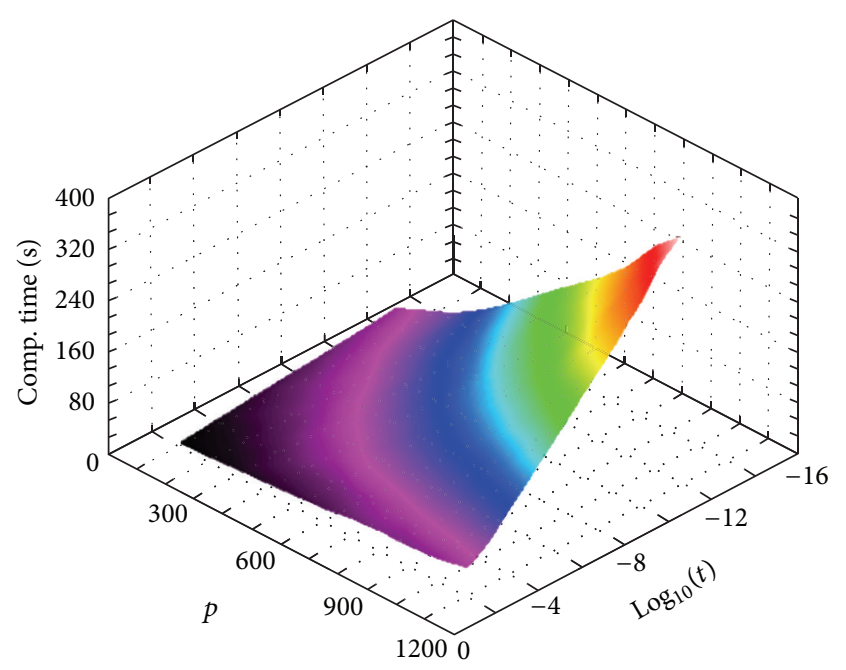

FIgure 2: Sensitivity of computational time (sample plot).

The assessment of the influence of $t$ and $p$ on the modal identification results can provide effective hints to ensure accurate modal estimates or to reduce the computational time with little or no accuracy losses. To this aim, the simulated dataset has been processed by SOBI, and the identified modes in the range $0-5 \mathrm{~Hz}$ have been compared with the theoretical values for different settings of $t$ and $p$. The cumulative frequency scatter $J_{f}$ and the cumulative discrepancy between corresponding mode shapes $J_{s}$

$$
\begin{gathered}
J_{f}=\sum_{i=1}^{N_{m}}\left|\frac{f_{i}^{\mathrm{SOBI}}-f_{i}^{\mathrm{ref}}}{f_{i}^{\mathrm{ref}}}\right| \cdot 100 \\
J_{s}=\sum_{i=1}^{N_{m}}\left[1-\operatorname{MAC}\left(\left\{\phi_{i}^{\mathrm{SOBI}}\right\},\left\{\phi_{i}^{\mathrm{ref}}\right\}\right)\right] \cdot 100
\end{gathered}
$$

quantify the accuracy of the modal identification results. In (21) $N_{m}$ is the number of identified structural modes, while the MAC between theoretical and estimated mode shapes is computed as per its definition [34]. The plot of $J_{f}+J_{s}$ versus $p$ and $t$ for the simulated dataset is shown in Figure 3(a). It is possible to recognize that accuracy is mainly influenced by $p$, while a weak variation with $t$ can be observed. In particular, for $t$ not larger than $1 E-8$, results do not change anymore. This limit value for $t$ is expected to be data dependent. However, similar sensitivity analyses carried out on real datasets provided the same results. Even if it is impossible to extrapolate a general rule, the obtained results seem to confirm that this value can be assumed as reference to obtain very stable results. Moreover, the little effect of $t$ on the accuracy of estimates allows setting a threshold larger than $1 E-8$ in order to reduce the computational time. The obtained results suggest that $t$ can be increased up to $1 E-4$ with negligible effects on the obtained modal parameter estimates and relevant reduction of computational time, in particular when the number of measurement channels is large. Above that value the approximations start inducing major effects on the estimates. Concerning the effect of $p$ on accuracy,
Figure 3(a) shows that the overall accuracy first improves and then gets worse for increasing values of $p$. This is an effect of the trend of $J_{f}$ versus $p$, since $J_{s}$ monotonically and slowly decreases with increasing values of $p$ (even if for a single mode the $J_{s}$ versus $p$ plot could not be monotonic), as shown in Figure 3(b). This is consistent with the higher robustness of SOBI to noise with respect to other BSS methods based on second-order statistics, such as AMUSE [35]. This robustness is the result of the joint diagonalization of several time-shifted covariance matrices instead of the eigenvalue decomposition of a single covariance matrix evaluated at a certain time lag. On the other hand, the trend of $J_{f}$ is probably due to the fact that the larger the value of $p$, the larger the effect of the disturbance beyond a certain decay level. As a final result, an optimal setting of $p$ able to maximize the overall accuracy of modal estimates can be obtained from the minimum of the $J_{f}+J_{s}$ function.

Repeating the sensitivity analysis for different values of the sampling frequency, in order to reduce or increase the number of modes in the frequency range of interest, the trend of the $J_{f}+J_{s}$ function is kept, but the value of $p$ corresponding to its minimum changes, as expected. However, when the sampling frequency changes by a factor of $x$, the optimal setting for $p$ changes by the same factor. Attention has been therefore focused on the source associated with the fundamental mode, which is obviously present in all the analyses, and on the decay level associated with the optimal value of $p$. The decay level was found to be the same for different values of the sampling frequency. In particular, it was in the order of $10 \%$ of the maximum amplitude of the first cycle occurring at $\tau_{k}>0$ (for $\tau_{k}=0 R_{s s}$ is always equal to one). Thus, the time to observe a given amplitude decay rather than the number of samples is responsible for the accuracy of estimates. This is consistent with the change of the optimal setting of $p$ with the adopted sampling frequency and with the increasing effect of noise when $p$ becomes too large.

The analysis of the decay level associated with the optimal setting of $p$ seems to suggest the following rule for its evaluation. The optimal value of $p$ can be determined in a couple of iterations by applying SOBI for a generic value of $p$ and changing it in a way able to achieve a ratio of about $10 \%$ between the amplitudes of the last (depending on the value of $p$ ) and the first cycle (at $\tau_{k}>0$ ) of the source correlation associated with the fundamental mode (Figure 4).

This rule has been validated through its application to real datasets. Taking into account the influence of $t$ on the accuracy of modal parameter estimates, a value of $1 \mathrm{E}-8$ has been adopted in the analyses. The obtained modal identification results are reported in Table 2 in comparison with reference estimates. A very good agreement can be observed, confirming that the proposed rule makes an effective setting of the analysis parameters able to provide accurate outputonly modal identification results possible.

In order to validate further the proposed rule for the setting of $p$, the real datasets have been analyzed also for different values of $p$. The function $J_{f}+J_{s}$ versus $p$ (Figure 5) has been evaluated around the estimated optimal value of $p$. The reference values of the natural frequencies and mode shapes are those provided by the SSI method (however, 


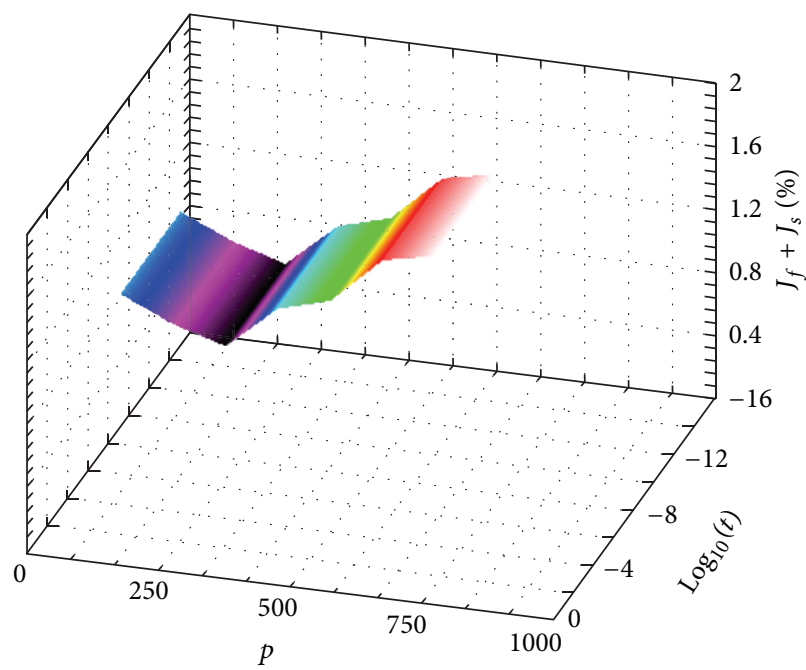

(a)

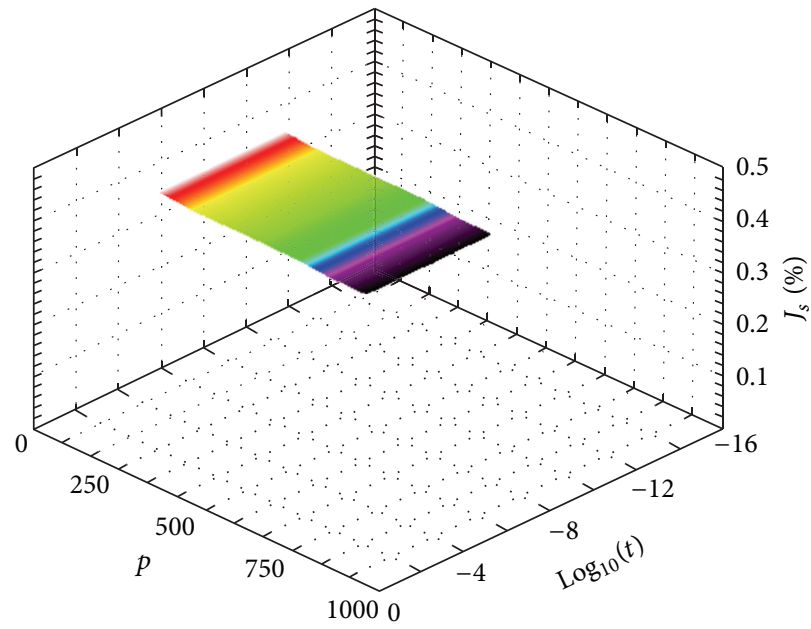

(b)

Figure 3: Sensitivity of overall accuracy (a) and mode shape accuracy (b) to $p$ and $t$.

TABLE 2: Test cases, modal identification results, and comparisons.

\begin{tabular}{|c|c|c|c|c|c|c|c|c|c|}
\hline Case study & Characteristics & $l$ & $\left(f_{s}[\mathrm{~Hz}], p\right)$ & Mode number & $f_{\text {SOBI }}[\mathrm{Hz}]$ & $f_{\text {ref }}[\mathrm{Hz}]$ & $\xi_{\text {SOBI }}[\%]$ & $\xi_{\text {ref }}[\%]$ & MAC \\
\hline \multirow{4}{*}{ 6-DOF system } & \multirow{4}{*}{ Simulated data } & \multirow{4}{*}{6} & \multirow{4}{*}{$(10,400)$} & $\mathrm{I}$ & 1.51 & 1.51 & 0.97 & 1.00 & 0.998 \\
\hline & & & & II & 2.82 & 2.82 & 0.73 & 0.80 & 0.999 \\
\hline & & & & III & 3.81 & 3.81 & 0.88 & 0.81 & 0.999 \\
\hline & & & & IV & 4.73 & 4.74 & 0.81 & 0.87 & 0.998 \\
\hline \multirow{3}{*}{$\begin{array}{l}\text { Tower of the Nations } \\
\text { (Naples) }\end{array}$} & \multirow{3}{*}{$\begin{array}{l}\text { Well-separated } \\
\text { modes }\end{array}$} & \multirow{3}{*}{8} & \multirow{3}{*}{$(5,360)$} & I & 0.81 & 0.81 & 0.36 & 0.40 & $\approx 1$ \\
\hline & & & & II & 1.38 & 1.38 & 0.97 & 1.17 & $\approx 1$ \\
\hline & & & & III & 1.66 & 1.66 & 0.69 & 0.63 & $\approx 1$ \\
\hline \multirow{3}{*}{$\begin{array}{l}\text { School of Engineering } \\
\text { (Naples) }\end{array}$} & \multirow{3}{*}{$\begin{array}{l}\text { Closely spaced } \\
\text { modes }\end{array}$} & \multirow{3}{*}{12} & \multirow{3}{*}{$(10,400)$} & $\mathrm{I}$ & 0.92 & 0.92 & 1.23 & 1.32 & $\approx 1$ \\
\hline & & & & II & 0.99 & 0.99 & 1.17 & 1.02 & 0.999 \\
\hline & & & & III & 1.30 & 1.30 & 0.58 & 0.64 & $\approx 1$ \\
\hline \multirow{2}{*}{$\begin{array}{l}\text { S. Maria del Carmine Bell } \\
\text { Tower (Naples) }\end{array}$} & \multirow{2}{*}{$\begin{array}{l}\text { Closely spaced } \\
\text { modes, noisy data }\end{array}$} & \multirow{2}{*}{12} & \multirow{2}{*}{$(5,300)$} & I & 0.70 & 0.70 & 0.96 & 0.92 & $\approx 1$ \\
\hline & & & & II & 0.76 & 0.76 & 0.92 & 0.83 & $\approx 1$ \\
\hline
\end{tabular}

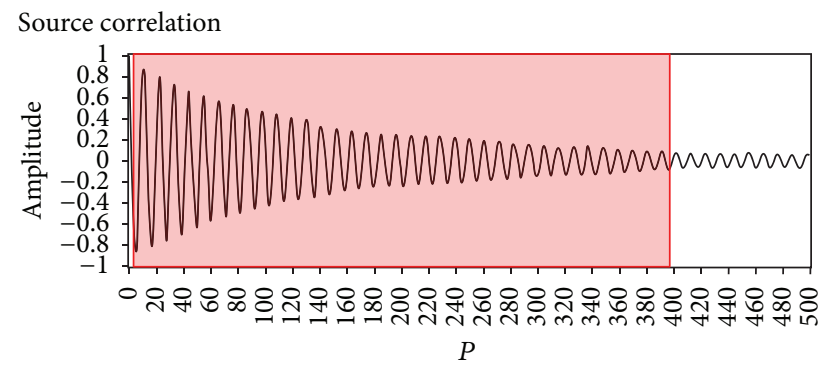

FIgURE 4: Illustration of the rule of thumbs for setting of $p$.

similar results have been obtained considering as reference estimates those provided by the FDD method). Figure 5 shows that the proposed rule is able to properly evaluate the optimal setting of $p$ even in the case of real datasets.

The proposed approach for the optimal setting of $p$ can easily be automated and used for the development of
SOBI-based automated OMA procedures for vibration-based SHM. It is worth pointing out that the possibility of automatically setting the analysis parameters without any preliminary calibration is a fundamental requirement for the development of automated OMA procedures. An effective control of computational efforts is possible by appropriate setting of $t$, taking into account that it negatively affects the accuracy of modal parameter estimates beyond the limit value of $1 E-$ 4. Thus, the present paper provides a contribution towards the development of innovative automated OMA procedures able to satisfy widely accepted target criteria reported in the literature $[6,7,9]$. However, automated OMA based on SOBI is out of the scope of the paper.

\section{Conclusions}

In the present paper the applicative perspectives of SOBI for OMA of civil structures have been discussed. Attention has been focused on SOBI because of its interesting performance, 


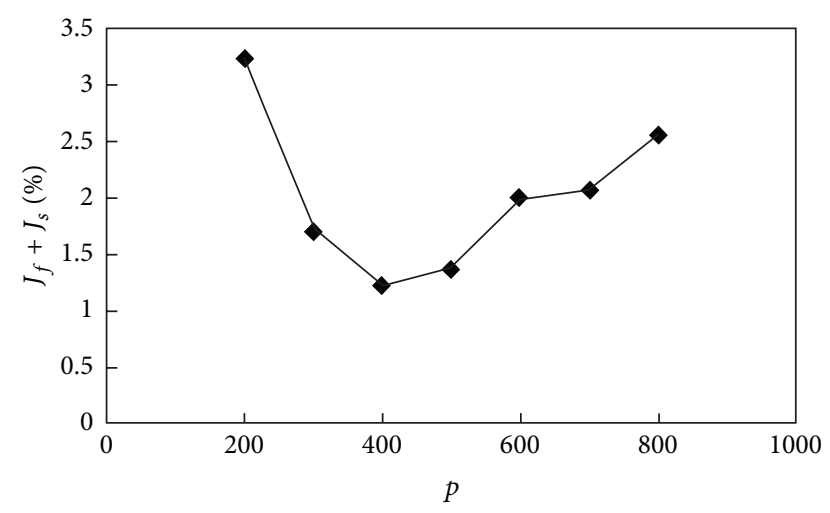

FIGURE 5: Validation of the proposed rule for setting of $p\left(t=10^{-8}\right.$, real dataset: "School of Engineering in Naples").

among the BSS methods, in the field of output-only modal identification and vibration-based SHM. In fact, the moderate complexity and computational demand of the algorithm and the separation of modal contributions make it suitable for automation and integration into SHM systems. In this perspective the influence of the JAD parameters on accuracy of modal estimates and computational time has been analyzed. The performed sensitivity analyses have made the definition of rules of thumbs for their optimal setting possible. These rules have also been validated against real datasets. The selected real case studies were representative of the different degree of complexity of modal identification test cases usually encountered in civil engineering. The good agreement between the modal property estimates provided by SOBI and those obtained by well-established OMA techniques, such as FDD and SSI, confirms SOBI as a valid alternative for outputonly modal identification of civil structures. The results of the analyses reported in this paper have also provided the basis for the rational development of SOBI-based automated OMA procedures able to provide accurate estimates and effective control of computational efforts. However, the development of automated OMA procedures based on SOBI is out of the scope of the paper. It will be the object of future studies.

\section{Conflict of Interests}

The author declares that there is no conflict of interests regarding the publication of this paper.

\section{References}

[1] C. Rainieri and G. Fabbrocino, Operational Modal Analysis of Civil Engineering Structures-An Introduction and Guide for Applications, Springer, New York, NY, USA, 2014.

[2] C. Rainieri, G. Fabbrocino, and E. Cosenza, "Some remarks on experimental estimation of damping for seismic design of civil constructions," Shock and Vibration, vol. 17, no. 4-5, pp. 383-395, 2010.

[3] E. Reynders, R. Pintelon, and G. de Roeck, "Uncertainty bounds on modal parameters obtained from stochastic subspace identification," Mechanical Systems and Signal Processing, vol. 22, no. 4, pp. 948-969, 2008.
[4] B. Cauberghe, P. Guillaume, P. Verboven, S. Vanlanduit, and E. Parloo, "On the influence of the parameter constraint on the stability of the poles and the discrimination capabilities of the stabilisation diagrams," Mechanical Systems and Signal Processing, vol. 19, no. 5, pp. 989-1014, 2005.

[5] C. Rainieri, G. Fabbrocino, and E. Cosenza, "On damping experimental estimation," in Proceedings of the 10th International Conference on Computational Structures Technology, Valencia, Spain, 2010.

[6] E. Reynders, J. Houbrechts, and G. de Roeck, "Fully automated (operational) modal analysis," Mechanical Systems and Signal Processing, vol. 29, pp. 228-250, 2012.

[7] F. Magalhães, A. Cunha, and E. Caetano, "Online automatic identification of the modal parameters of a long span arch bridge," Mechanical Systems and Signal Processing, vol. 23, no. 2, pp. 316-329, 2009.

[8] R. Brincker, P. Andersen, and N. J. Jacobsen, "Automated frequency domain decomposition for operational modal analysis," in Proceedings of the 25th SEM International Modal Analysis Conference, Orlando, Fla, USA, 2007.

[9] C. Rainieri and G. Fabbrocino, "Automated output-only dynamic identification of civil engineering structures," Mechanical Systems and Signal Processing, vol. 24, no. 3, pp. 678-695, 2010.

[10] C. Rainieri, G. Fabbrocino, and E. Cosenza, "Near real-time tracking of dynamic properties for standalone structural health monitoring systems," Mechanical Systems and Signal Processing, vol. 25, no. 8, pp. 3010-3026, 2011.

[11] C. Zang, M. I. Friswell, and M. Imregun, "Structural damage detection using independent component analysis," Structural Health Monitoring, vol. 3, no. 1, pp. 69-83, 2004.

[12] M. J. Roan, J. G. Erling, and L. H. Sibul, "A new, nonlinear, adaptive, blind source separation approach to gear tooth failure detection and analysis," Mechanical Systems and Signal Processing, vol. 16, no. 5, pp. 719-740, 2002.

[13] J. Antoni, L. Garibaldi, S. Marchesiello, and M. Sidhamed, "New separation techniques for output-only modal analysis," Shock and Vibration, vol. 11, no. 3-4, pp. 227-242, 2004.

[14] J. Antoni, "Blind separation of vibration components: principles and demonstrations," Mechanical Systems and Signal Processing, vol. 19, no. 6, pp. 1166-1180, 2005.

[15] G. Kerschen, F. Poncelet, and J. C. Golinval, "Physical interpretation of independent component analysis in structural dynamics," Mechanical Systems and Signal Processing, vol. 21, no. 4, pp. 1561-1575, 2007.

[16] B. Ans, J. Hérault, and C. Jutten, "Adaptive neural architectures: detection of primitives," in Proceedings of the COGNITIVA '85, pp. 593-597, Paris, France, June 1985.

[17] A. Belouchrani, K. Abed-Meraim, J. Cardoso, and E. Moulines, "A blind source separation technique using second-order statistics," IEEE Transactions on Signal Processing, vol. 45, no. 2, pp. 434-444, 1997.

[18] J. Storck and G. Deco, "Nonlinear independent component analysis and multivariate time series analysis," Physica D: Nonlinear Phenomena, vol. 108, no. 4, pp. 335-349, 1997.

[19] H. N. Thi and C. Jutten, "Blind source separation for convolutive mixtures," Signal Processing, vol. 45, no. 2, pp. 209-229, 1995.

[20] F. Poncelet, G. Kerschen, J. Golinval, and D. Verhelst, "Outputonly modal analysis using blind source separation techniques," Mechanical Systems and Signal Processing, vol. 21, no. 6, pp. 2335-2358, 2007. 
[21] W. Zhou and D. Chelidze, "Blind source separation based vibration mode identification," Mechanical Systems and Signal Processing, vol. 21, no. 8, pp. 3072-3087, 2007.

[22] S. I. McNeill and D. C. Zimmerman, "A framework for blind modal identification using joint approximate diagonalization," Mechanical Systems and Signal Processing, vol. 22, no. 7, pp. 1526-1548, 2008.

[23] R. Brincker and J. A. Larsen, "Obtaining and estimating low noise floors in vibration sensors," in Proceedings of the 25th SEM International Modal Analysis Conference, Orlando, Fla, USA, 2007.

[24] A. Belouchrani and A. Cichocki, "Robust whitening procedure in blind source separation context," Electronics Letters, vol. 36, no. 24, pp. 2050-2051, 2000.

[25] J. F. Cardoso and A. Souloumiac, "Jacobi angles for simultaneous diagonalization," SIAM Journal on Matrix Analysis and Applications, vol. 17, no. 1, pp. 161-164, 1996.

[26] C. Rainieri and G. Fabbrocino, "Performance assessment of selected oma techniques for dynamic identification of geotechnical systems and closely spaced structural modes," Journal of Theoretical and Applied Mechanics, vol. 49, no. 3, pp. 825-839, 2011.

[27] S. Gade, N. B. Møller, H. Herlufsen, and H. Konstantin-Hansen, "Frequency domain techniques for operational modal analysis," in Proceedings of the 1st International Operational Modal Analysis Conference, pp. 261-271, Copenhagen, Denmark, 2005.

[28] C. Rainieri, G. Fabbrocino, and G. M. Verderame, "Nondestructive characterization and dynamic identification of a modern heritage building for serviceability seismic analyses," NDT and E International, vol. 60, pp. 17-31, 2013.

[29] C. Rainieri, G. Fabbrocino, and E. Cosenza, "Integrated seismic early warning and structural health monitoring of critical civil infrastructures in seismically prone areas," Structural Health Monitoring, vol. 10, no. 3, pp. 291-308, 2011.

[30] C. Rainieri and G. Fabbrocino, "Operational modal analysis for the characterization of heritage structures," Geofizika, vol. 28, no. 1, pp. 109-126, 2011.

[31] R. Brincker, L. M. Zhang, and P. Andersen, "Modal identification of output-only systems using frequency domain decomposition," Smart Materials and Structures, vol. 10, no. 3, pp. 441445, 2001.

[32] P. van Overschee and B. de Moor, Subspace Identification for Linear Systems: Theory-Implementation-Applications, Kluwer Academic, Dordrecht, The Netherlands, 1996.

[33] B. Hazra, Hybrid time and time-frequency blind source separation towards ambient system identification of structures [Ph.D. thesis], University of Waterloo, Waterloo, Canada, 2010.

[34] R. J. Allemang and D. L. Brown, "A correlation coefficient for modal vector analysis," in Proceedings of the 1st SEM International Modal Analysis Conference, Orlando, Fla, USA, 1982.

[35] L. Tong, R. W. Liu, V. C. Soon, and Y. F. Huang, "Indeterminacy and identifiability of blind identification," IEEE Transactions on Circuits and Systems, vol. 38, no. 5, pp. 499-509, 1991. 

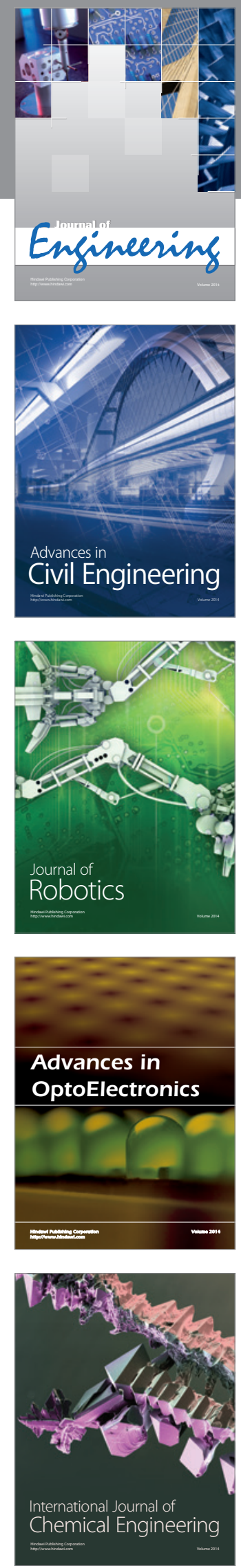

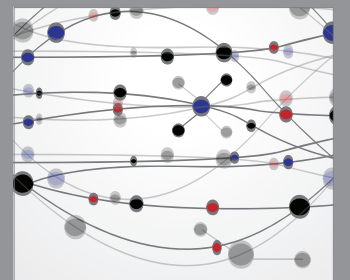

The Scientific World Journal
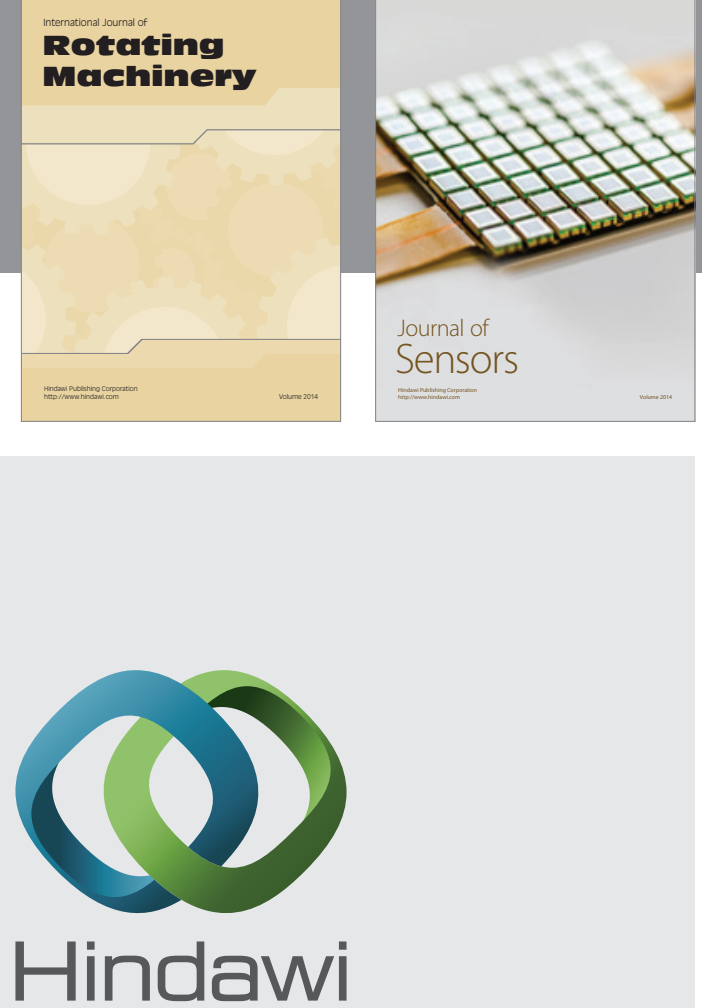

Submit your manuscripts at http://www.hindawi.com
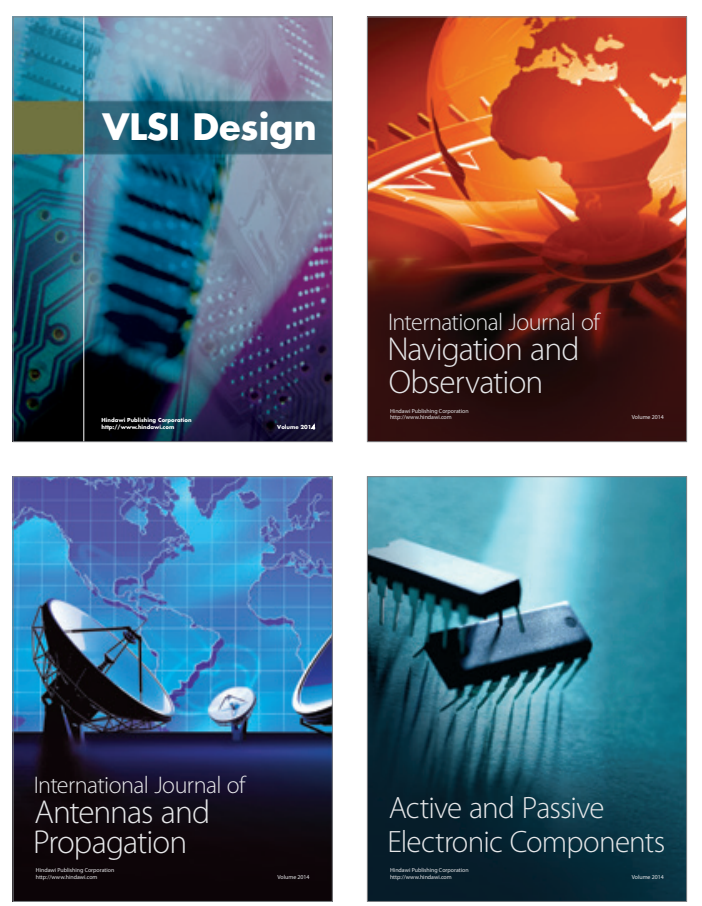
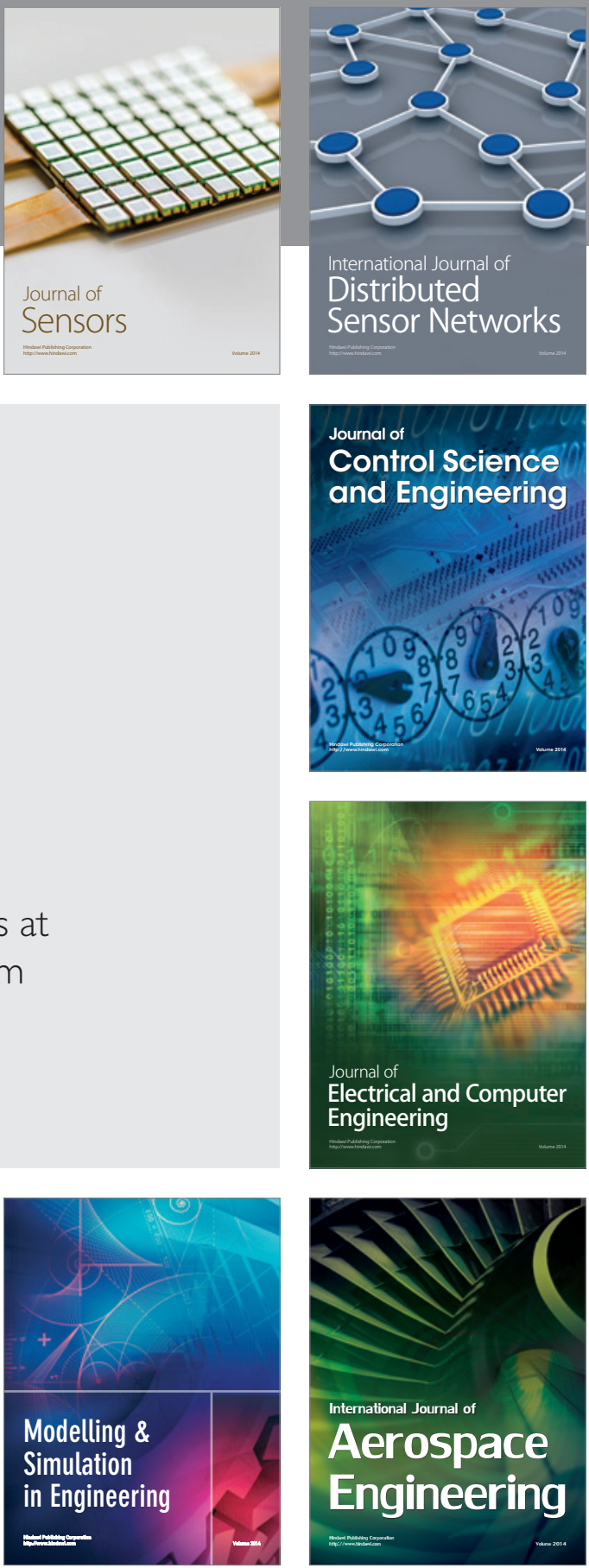

Journal of

Control Science

and Engineering
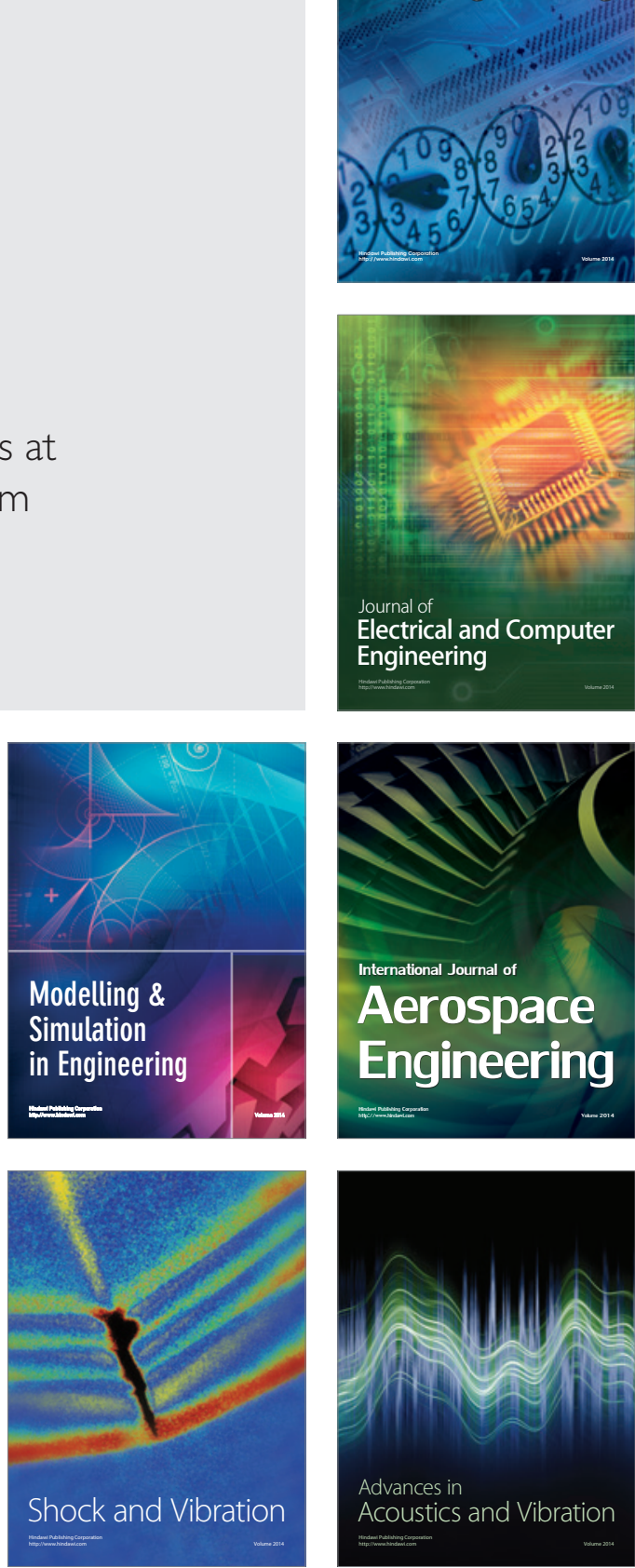